\title{
PROBABILIDADE: UMA VISÃO OLÍMPICA
}

Probability: an olympic vision

\author{
RAFAEL ARAUJO DE SOUZA ${ }^{1}$ \\ DAIANA CARVALHO DUTRA ${ }^{2}$
}

\begin{abstract}
RESUMO: Apresenta-se, neste trabalho, o resultado de uma pesquisa do tipo bibliográfica sobre probabilidade, buscando apresentar uma visão olímpica, por meio de uma série de problemas de olimpíadas de matemática, como a OBMEP, OBM e o Canguru de Matemática, mostrando como é possível ensinar probabilidade aplicando problemas olímpicos, despertando cada vez mais o interesse dos alunos por competições matemáticas, além também de tirar a ideia de que o que cai nas olimpíadas de matemática não é o que é visto em sala de aula. Este artigo está dividido em quatro partes: na primeira parte é apresentado uma pequena descrição sobre algumas competições de matemática. Na segunda, tem-se os primeiros passos em probabilidade, onde se apresenta a definição clássica de probabilidade, aplicando-a em problemas olímpicos. Na terceira, é abordado o espaço de probabilidades e na quarta, trata-se da independência de eventos, que é a parte mais importante do artigo, onde se aplica os conceitos discutidos em diversos problemas olímpicos.
\end{abstract}

Palavras-chaves: Probabilidade. Olimpíadas. Matemática.

\begin{abstract}
This paper presents the result of a bibliographic research on probability, seeking to present an Olympic vision, through a series of mathematical Olympiad problems, such as OBMEP, OBM and Mathematics Kangaroo, showing how it is It is possible to teach probability by applying Olympic problems, increasingly arousing students' interest in mathematical competitions, and also the idea that what falls in the math Olympics is not what is seen in the classroom. This article is divided into four parts: The first part gives a short description of some math competitions. In the second, there are the first steps in probability, which presents the classic definition of probability, applying it to Olympic problems. The third deals with the probability space and the fourth deals with the independence of events, which is the most important part of the article, where the concepts discussed in various Olympic problems are applied
\end{abstract}

Key-words: Probability. Olympics. Mathematics.

\footnotetext{
${ }^{1}$ Professor da Secretaria de Estado da Educação do Piauí (SEDUC-PI), graduado em Matemática (UFPI), Especialista em Metodologia do Ensino da Matemática (FAERPI) e em Matemática Financeira e Estatística (FAVENI). E- mail: rafaelmat13@gmail.com

2 Professora da Secretaria de Estado da Educação do Piauí (SEDUC-PI), graduada em Matemática (UFPI), Especialista em Metodologia do Ensino da Matemática (FAERPI). E-mail: daiana10carvalho@hotmail.com
} 


\section{INTRODUÇÃO}

Quando se fala de probabilidade, logo vem em mente o nome do italiano Gerolamo Cardano (1501 - 1576), no qual foi o primeiro a fazer registros ligados a teoria da probabilidade, no caso, sobre jogos de azar.

Aproximadamente cem anos depois, um impulso ao desenvolvimento da teoria das probabilidades foi dado por Blaise Pascal, através de trocas de cartas com Pierre de Fermat (1601 - 1665), onde discutiam problemas sobre jogos.

Só em 1713 que o primeiro artigo completo sobre probabilidade foi escrito, no caso, por Jacques Bernouli, em sua obra Ars Conjectandi (Arte de Conjecturar), onde daí em diante, outros matemáticos deram e veem dando suas contribuições ao desenvolvimento da teoria das probabilidades.

Em sua obra, IEZZI et al. nos diz o que a teoria da probabilidade nos permite.

A teoria da probabilidade permite que se façam previsões sobre as chances de um acontecimento ocorrer, em certo experimento aleatório, a partir da análise dos resultados obtidos, quando esse experimento é repetido, nas mesmas condições, um grande número de vezes. (IEZZI et al., 2016, p.252)

A probabilidade, como citado acima, nos permite prevê chances de algum acontecimento ocorrer, de um determinado experimento aleatório. Logo os experimentos estudados precisam ser aleatórios, que segundo Morgado et al. (1991, p.128), “os experimentos que repetidos sob as mesmas condições produzem resultados geralmente diferentes são chamados de experimentos aleatórios."

Na educação básica, este tópico não é dado de forma que provoque os alunos a pensarem, isso quando é dada, pois a probabilidade é apresentada de forma bem mecânica, sem que provoque os alunos a pensarem de forma criativa, o que torna as aulas mais chatas e até mesmo sem o interesse dos alunos a participarem.

Sendo um dos conteúdos mais complicados para os alunos, visto que esse tópico requer uma boa dose de criatividade, sem falar que sempre há a dúvida: "Quando somo e quando multiplico?” Em sala de aula, essa parte da matemática por um lado é esquecida e não é dada e por outro lado ela é deixada de lado simplesmente pelo fato do professor não ter afinidade e também não domina-la bem, o que é errado, pois a probabilidade está presente constantemente em olimpíadas de matemática no Enem e no ensino superior. 
Uma forma de trabalhar a criatividade dos alunos é trabalhar com problemas olímpicos, que além de instigar os alunos, motiva-os a participar de competições matemáticas que a cada ano que passa, veem ganhando espaço e aos medalhistas tendo a chance de concorrer a vagas olímpicas em faculdades de renome no Brasil.

Uma metodologia bem interessante e que pode ser utilizada em sala de aula é a resolução de problemas, como diz Freitas et. al. (2014, p.3) “Ao observarmos algumas das principais tendências atuais na área de Educação Matemática, constatamos que a resolução de problemas aparece frequentemente como "motor" de muitas propostas inovadoras.", o que pode ser associado com problemas olímpicos, que são desafiadores aos alunos e faz com que eles fiquem intrigados a resolvê-los.

Frente a este contexto, o presente trabalho tem como intuito trazer a tona a importância da utilização de problemas olímpicos para o ensino de probabilidade, além de mostrar que é possível preparar todo o curso em cima destas competições.

Com o propósito descrito acima, este trabalho foi organizado da seguinte maneira. A primeira seção é apresentada uma pequena descrição sobre algumas competições de matemática.

$\mathrm{Na}$ segunda seção tem-se os primeiros passos em probabilidade, onde se apresenta a definição clássica e aplicada em alguns problemas olímpicos.

$\mathrm{Na}$ terceira seção versa sobre o espaço de probabilidades, onde é apresentado algumas propriedades interessantes e importantes da probabilidade

E por fim, na quarta seção apresenta-se o principal tópico, no caso, a independência de eventos, que nos permitirá resolver vários problemas olímpicos, usando todos os conteúdos trabalhados nas seções anteriores.

\section{OLIMPIADAS DE MATEMÁTICA}

Nos dias de hoje os alunos apaixonados por matemática, especialmente em competições, possuem uma gama de oportunidades com a grande diversidade de competições, estaduais, nacionais e internacionais. Faremos uma pequena listagem e descrição sobre algumas competições. Todos as informações aqui estão nas referências [2], [3], [4] e [11].

O Concurso Canguru de Matemática é uma competição internacional que ocorre anualmente e é destinada a todos os alunos da educação básica $\left(3^{\circ}\right.$ ano do ensino fundamental até o $3^{\circ}$ ano do ensino médio). É considerada a maior competição internacional do mundo, com uma média superior a 6 milhões de participantes anualmente, presente em 75 países. 
A OBMEP é uma competição de nível nacional, criada em 2005 com o objetivo de estimular o estudo da matemática e identificar talentos na área. É um projeto nacional direcionado a princípio às escolas públicas, e atualmente também às escolas privadas brasileiras. A realização é de responsabilidade do Instituto Nacional de Matemática Pura e Aplicada - IMPA, com o apoio da Sociedade Brasileira de Matemática - SBM, sendo que é promovida com recursos do Ministério da Educação e do Ministério da Ciência, Tecnologia, Inovações e Comunicações - MCTIC. é um projeto nacional dirigido às escolas públicas e privadas brasileiras, realizado pelo Instituto Nacional de Matemática Pura e Aplicada - IMPA, com o apoio da Sociedade Brasileira de Matemática SBM, e promovida com recursos do Ministério da Educação e do Ministério da Ciência, Tecnologia, Inovações e Comunicações - MCTIC.

A OBM é uma competição para estudantes da educação básica (fundamental maior e ensino médio) e do ensino superior de instituições públicas e privadas do Brasil; é uma realização conjunta da Sociedade Brasileira de Matemática (SBM) e do Instituto de Matemática Pura e Aplicada (IMPA). Sua coordenação está a cargo da comissão gestora e da comissão nacional de olimpíadas de matemática da SBM.

A partir de 2017 teve seu formato mudado, agora nos níveis 1, 2 e 3 estando integrada OBMEP, havendo apenas uma fase no segundo semestre com alunos convidados, mediante ótimos desempenhos na OBMEP e olimpíadas de matemática regionais apoiadas pela OBM. Já no nível universitário, a mudança está no fato da inscrição ser agora individual.

Dentre os objetivos da OBM, podemos citar alguns como selecionar os estudantes que representarão o Brasil e competições internacionais de matemática a partir do seu desempenho na OBM, realizando seu devido treinamento; apoiar as competições regionais de matemática em todo Brasil e organizar diversas competições internacionais de matemática, quando realizadas no Brasil. Os demais objetivos são os mesmos da OPIM, mas com caráter nacional.

Existem outras diversas competições de matemática, nos níveis estaduais, como por exemplo a OPIM (Olimpíada Piauiense de Matemática), nacionais como a OBMV (Olimpíada Brasileira Virtual de Matemática) e internacionais como a OIMSF (Olimpíada Internacional Matemática Sem Fronteiras).

Como podemos ver, existem muitas oportunidades para os alunos se divertirem estudando e competindo de forma saudável, precisando apenas que nós professores apesentemos esse mundo de diversão para os alunos. 


\section{PRIMEIROS PASSOS EM PROBABILIDADE}

Uma das principais aplicações dos métodos de contagem, consiste na resolução de problemas simples de probabilidades de eventos aleatórios, eventos estes, que quando repetidos sob as mesmas condições produzem resultados geralmente diferentes. Sendo assim, é preciso contar, como veremos a seguir, os resultados favoráveis e os possíveis. Vale salientar ainda que, os estudos das probabilidades têm suas origens nos jogos de azar. Neste primeiro momento daremos os primeiros passos em probabilidade, com a definição clássica, aplicando-a em alguns problemas olímpicos. Todos os resultados aqui discutidos estão associados as referências [2], [4] e [5].

Exemplo 1. Ao lançar um dado honesto de seis faces, qual a probabilidade de sair um número múltiplo de 3 ?

Solução: Observe que temos os seguintes resultados possíveis

$$
\Omega=\{1,2,3,4,5,6\}
$$

Nota-se que todos os possíveis resultados têm a mesma chance de ocorrer, no caso, aproximadamente $\frac{1}{6}$ dos lançamentos. Sendo assim, dizemos que cada uma das faces tem probabilidade de $\frac{1}{6}$ de sair. Como os múltiplos de 3 são apenas o próprio 3 e o 6 , então a probabilidade de sair um múltiplo de 3 é de

$$
\frac{1}{6}+\frac{1}{6}=\frac{2}{6}=\frac{1}{3}
$$

Em um de seus trabalhos, Carvalho nos traz uma generalização teórica para este exemplo, a saber:

De um modo geral, quando todos os resultados têm a mesma chance de ocorrer, a probabilidade de um evento é a razão entre o número de resultados relativos ao evento e o número total de resultados. Em outras palavras, é a razão entre o número de casos favoráveis à ocorrência do evento e o número total de casos. (CARVALHO, 2015, p.25)

Definição 1: Define-se a probabilidade de um subconjunto $A \subset \Omega$ como sendo o número

$$
P(A)=\frac{|A|}{|\Omega|}
$$

Onde $|A|$ representa o número de resultados favoráveis e $|\Omega|$ o número de resultados possíveis. Define-se também o subconjunto de todos os resultados possíveis de Espaço Amostral e um subconjunto $A$ de $\Omega$ de Evento.

Problema Olímpico 1. (OBMEP - 2005) Brasil e Argentina participam de um campeonato internacional de futebol no qual competem oito seleções. Na primeira rodada serão realizadas quatro partidas, nas quais os adversários são escolhidos por sorteio. Qual é a probabilidade de Brasil e Argentina se enfrentarem na primeira rodada? 
Solução: Fixando o Brasil, há apenas 7 países que podem o enfrentar na primeira rodada, sendo a Argentina um deles, com isso o problema resume-se apenas em calcular a probabilidade de ser sorteado a Argentina como seu adversário inicial. Sendo $|A|=1$ e $|\Omega|=7$, tem-se que $P(A)=\frac{1}{7}$. Portanto, a probabilidade de Brasil e Argentina se enfrentarem na primeira rodada é de $\frac{1}{7}$.

Problema Olímpico 2. (OBMEP - 2016) A professora decidiu premiar, por sorteio, dois dentre os 20 alunos da turma de João. Para o sorteio, 20 bolas com os números dos alunos foram colocadas em uma caixa. A primeira bola sorteada pela professora caiu no chão e se perdeu, sem que ninguém visse seu número. Ela decidiu fazer o sorteio com as bolas restantes. Qual é a probabilidade de que João tenha sido um dos dois alunos sorteados?

Figura 1: Mesa

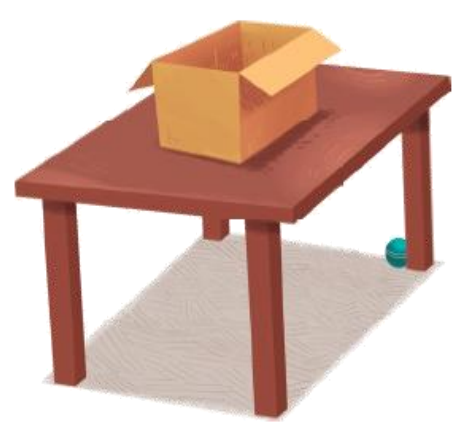

Fonte: OBMEP (2016)

Solução: Para resolver este problema, como não sabemos o número que estava na bola que caiu, podemos considerar normalmente como se a bola não houvesse caído. Sendo assim, resta descobrirmos o número de casos favoráveis $|A|$ e os casos possíveis $|\Omega|$ (espaço amostral), no qual usaremos os métodos de contagem.

Os resultados favoráveis são os conjuntos de duas bolas sorteadas onde o número de João está em uma delas, logo, segundo o Princípio Fundamental da Contagem temos: $|A|=1 \cdot 19$, pois é suficiente escolher apenas o número da outra bola.

Já os resultados possíveis, no caso o espaço amostral, precisamos contar quantas combinações de duas bolas são possíveis, a saber: $|\Omega|=\left(\begin{array}{c}20 \\ 2\end{array}\right)=\frac{20 !}{2 ! \cdot(20-2) !}=10 \cdot 19$. Portanto, a probabilidade da bola com o número de João ser sorteada é:

$$
P(A)=\frac{1 \cdot 19}{10 \cdot 19}=\frac{1}{10}
$$

Problema Olímpico 3. (Canguru de Matemática - 2017) Num dado cúbico especial, os números escritos nas faces são $-3,-2,-1,0,1,2$. Se o dado for lançado duas vezes e os números obtidos multiplicados, qual é a probabilidade que o produto seja negativo?

Solução: Vejamos o diagrama abaixo 
Figura 2: Diagrama de Possibilidades

\begin{tabular}{|c|c|c|c|c|c|c|c|}
\hline & \multicolumn{6}{|c|}{ Número do segundo lançamento } \\
\hline & & -3 & -2 & -1 & $\mathbf{0}$ & $\mathbf{1}$ & 2 \\
\hline \multirow{6}{*}{ 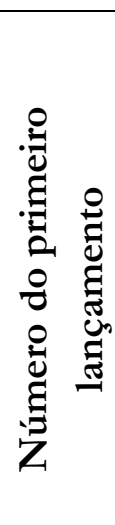 } & -3 & $(+)$ & $(+)$ & $(+)$ & (0) & $(-)$ & $(-)$ \\
\hline & -2 & $(+)$ & $(+)$ & $(+)$ & (0) & $(-)$ & $(-)$ \\
\hline & -1 & $(+)$ & $(+)$ & $(+)$ & (0) & $(-)$ & $(-)$ \\
\hline & $\mathbf{0}$ & (0) & (0) & (0) & (0) & (0) & (0) \\
\hline & 1 & $(-)$ & $(-)$ & $(-)$ & (0) & $(+)$ & $(+)$ \\
\hline & 2 & $(-)$ & $(-)$ & $(-)$ & (0) & $(+)$ & $(+)$ \\
\hline
\end{tabular}

Fonte: Autor (2020)

Como sabe-se, para o produto de dois números ser negativo é preciso que eles tenham sinais diferentes, logo, o quadro acima está mapeando todas as possibilidades, restando apenas contar os resultados favoráveis e possíveis. Sendo assim tem-se $|A|=12$ e $|\Omega|=36$. Portanto a probabilidade que o produto seja negativo é:

$$
P(A)=\frac{12}{36}=\frac{1}{3}
$$

Vale salientar que estes problemas acima trabalhados, com uma boa bagagem dos assuntos podem ser resolvidos de outra maneira, mais rápida e até mais sofisticada. Sendo assim, mais adiante retornaremos a eles para uma resolução alternativa, a medida que o estudo for sendo desenvolvido.

\section{ESPAÇO DE PROBABILIDADE}

A seguir será apresentada uma noção geral de probabilidade, além de algumas consequências e propriedades, fazendo pequenas demonstrações e aplicando-as sempre em problemas olímpicos. Todos os resultados aqui discutidos estão associados as referências [1], [7], [8] e [9].

Definição 2: Seja $\Omega$ um espaço amostral (conjunto). Uma função $P$ definida para todos os subconjuntos de $\Omega$ (chamados eventos) é chamada uma probabilidade se

i) $\quad 0 \leq P(A) \leq 1$, para todo evento $A \subset \Omega$;

ii) $P(\varnothing)=0, P(\Omega)=1$;

Cadernos Cajuína, V. 5, N. 1, 2020, p. 148-164. 
iii) Se $A$ e $B$ são eventos disjuntos ${ }^{3}$ (também chamados de mutuamente exclusivos), então $P(A \cup B)=P(A)+P(B)$.

Em alguns casos, calcular a probabilidade de um determinado evento não ocorrer é bem mais simples do que calcular a probabilidade que ele ocorra. A seguir apresentaremos a ferramenta na qual utilizaremos nestes casos, chamada de probabilidade do complementar.

Proposição 1: A probabilidade de não ocorrer o evento A de um espaço amostral $\Omega$ é dada por

$$
P\left(A^{c}\right)=1-P(A)
$$

Em que $A^{c}$ é o complementar de A em relação à $\Omega$, ou o evento "não ocorrer A".

Demonstração: Sabemos que

$$
1=P(\Omega)=\mathrm{P}\left(\mathrm{A} \cup A^{c}\right)=\mathrm{P}(\mathrm{A})+\mathrm{P}\left(A^{c}\right) .
$$

Portanto,

$$
P\left(A^{c}\right)=1-P(A)
$$

Proposição 2: A probabilidade de ocorrer o evento A ou o evento B é igual a probabilidade de ocorrer A mais a probabilidade de ocorrer B menos a probabilidade de ocorrer A e B, ou seja:

$$
P(A \cup B)=P(A)+P(B)-P(A \cap B) .
$$

Observe que quando $A$ e $B$ são eventos disjuntos, a Proposição 2 cai na condição iii) da Definição 2. Vejamos uma simples demonstração.

Demonstração: Sejam $A$ e $B$ eventos equiprováveis de um mesmo espaço amostral finito e não vazio $\Omega$. Como $A \cap B$ representa os elementos que satisfazem simultaneamente a $A$ e $B$ e $A \cup B$ os elementos que satisfazem a $\mathrm{A}$ ou $\mathrm{B}$, tem-se que:

$$
|A \cup B|=|A|+|B|-|A \cap B| .4
$$

Neste caso, dividindo os dois lados da igualdade da equação acima por $|\Omega|$, temos:

$$
\frac{|A \cup B|}{|\Omega|}=\frac{|A|}{|\Omega|}+\frac{|B|}{|\Omega|}-\frac{|A \cap B|}{|\Omega|} \Rightarrow P(A \cup B)=P(A)+P(B)-P(A \cap B) .
$$

\footnotetext{
${ }^{3} A$ e $B$ são disjuntos quando não têm elementos em comum ou seja $A \cap B=\emptyset$.

${ }^{4}$ Quando A e B são conjuntos finitos, tem-se: $|A \cup B|=|A|+|B|-|A \cap B|$
} 
Este resultado é conhecido como a probabilidade da união de dois conjuntos, no entanto, nos restringimos a dois conjuntos neste artigo, porém pode-se generalizar para uma quantidade finita n de conjuntos, fazendo uma versão do Princípio da Inclusão-Exclusão para probabilidades. Para mais detalhes sobre essa generalização vide [7].

Vejamos um exemplo.

Exemplo 2. Um número entre 1 e 200 é escolhido aleatoriamente. Qual a probabilidade de que seja divisível por 5 ou 7 ?

Solução: A princípio, note que o conectivo "ou" indica que o problema se trata da probabilidade da união de dois ou mais eventos. Logo, sejam os eventos $A$ e $B$ definidos por "Ser divisível por 5"e "Ser divisível por 7", respectivamente e $A \cap B$ o evento "ser divisível por 5 e 7 (por 35)" temse:

$$
\begin{aligned}
& P(A)=\frac{\left\lfloor\frac{200}{5}\right\rfloor}{200}=\frac{40}{200} \\
& P(B)=\frac{\left\lfloor\frac{200}{7}\right\rfloor}{200}=\frac{28}{200} \\
& P(A)=\frac{\left\lfloor\frac{200}{35}\right\rfloor}{200}=\frac{5}{200}
\end{aligned}
$$

Sendo assim,

$$
P(A \cup B)=P(A)+P(B)-P(A \cap B)=\frac{40}{200}+\frac{28}{200}-\frac{5}{200}=\frac{63}{200} .
$$

Observação: Aqui usamos a função menor inteiro \ 」, que nos dará a quantidade de divisores de um determinado número dentro de um intervalo definido.

\section{INDEPENDÊNCIA DE EVENTOS}

Nesta seção trabalharemos com um dos principais pontos da probabilidade, a Independência de Eventos, o que nos ajudará a resolver a maioria dos problemas de probabilidade de olimpíadas de matemática, além de nos proporcionar agilidade em suas resoluções. Todos os resultados aqui discutidos estão associados as referências [2], [3], [4 ], [8] e [9]. 
Definição 3: Dados dois eventos A e B, a probabilidade condicional de A dado B é o número $P(A \cap B) / P(B)$. Representaremos este número pelo símbolo $P(A / B)$. Temos então:

$$
P(A / B)=\frac{P(A \cap B)}{P(B)}
$$

Observe que este número só estará definido caso $P(B)>0$. Além disso, uma forma alternativa de vermos a expressão acima, seria $P(A \cap B)=P(A / B) \cdot P(B)$.

Quando a ocorrência do evento $B$ não afeta a probabilidade de ocorrer o evento $A$, neste caso $P(A / B)=P(A)$, dizemos que os eventos $A$ e $B$ são independentes, $\operatorname{logo} P(A \cap B)=$ $P(A) \cdot P(B)$ como veremos na definição a seguir.

Definição 4: Dois eventos $A$ e $B$ são chamados independentes se

$$
P(A \cap B)=P(A) \cdot P(B)
$$

Como consequência imediata da definição temos que o vazio $\emptyset$ e o espaço amostral $\Omega$ são independentes de qualquer outro evento, a saber:

Seja $B$ um evento qualquer, logo

$$
\begin{gathered}
P(\varnothing \cap B)=P(\varnothing)=0=P(\varnothing) \cdot P(B) \\
P(\Omega \cap B)=P(B)=1 \cdot P(B)=P(\Omega) \cdot P(B)
\end{gathered}
$$

Vamos aplicar s conteúdos aprendidos em um exemplo para depois aplicar em problemas olímpicos.

Exemplo 3. probabilidade de um atirador X acertar um alvo é de $80 \%$, e a probabilidade de um atirador Y acertar o mesmo alvo é de 90\%.

(a) ambos atinjam o alvo?

(b) pelo menos um atinja o alvo?

Solução: (a) Neste problema é natural admitirmos a independência dos eventos, logo a probabilidade dos dois acertarem o alvo será:

$$
P(X \cap Y)=0,80 \cdot 0,90=0,72 \therefore 72 \%
$$

(b) Neste item vamos usar a ideia do complementar, ou seja, calcular a probabilidade de ambos não acertarem o alvo e depois, calculamos o que se pede no item, que é pelo menos um acertar o alvo. Sendo assim, 


$$
P\left(X^{c} \cap Y^{c}\right)=0,20 \cdot 0,10=0,02 .
$$

Logo a probabilidade de pelo menos um acertar o alvo será de

$$
1-0,02=0,98 \therefore 98 \%
$$

A noção de independência para $n$ eventos, ocorre de forma natural partindo de alguns resultados omitidos aqui. Para um estudo mais a fundo vide [9]. Segue a definição generalizada.

Definição 5: $A_{1}, A_{2}, \ldots, A_{n}$ são independentes se $\forall k$, e $\forall i_{1}, i_{2}, \ldots, i_{k}$, tem-se:

$$
P\left(A_{i_{1}} \cap A_{i_{2}} \cap \ldots \cap A_{i_{k}}\right)=P\left(A_{i_{1}}\right) \cdot P\left(A_{i_{2}}\right) \ldots P\left(A_{i_{k}}\right) .
$$

Problema Olímpico 4. (Canguru de Matemática - 2014) Numa reserva ecológica há nove cangurus que são ou prateados ou dourados. Quando três desses cangurus se encontram ao acaso, a probabilidade de que nenhum deles seja prateado é igual a dois terços. Quantos deles são dourados?

Solução: Observe que temos 9 cangurus que são ou prateados ou dourados, logo seja x a quantidade de cangurus dourados. Sendo assim, temos

$$
\frac{x}{9} \cdot \frac{(x-1)}{8} \cdot \frac{(x-2)}{7}=\frac{2}{3} \Leftrightarrow x(x-1)(x-2)=8 \cdot 7 \cdot 6 \Leftrightarrow x=8,
$$

pois é a única solução inteira para a equação do $3^{\circ}$ grau acima. Portanto, número de cangurus dourados é 8.

Problema Olímpico 5. (OBM - 2006) Uma colônia de amebas tem inicialmente uma ameba amarela e uma ameba vermelha. Todo dia, uma única ameba se divide em duas amebas idênticas. Cada ameba na colônia tem a mesma probabilidade de se dividir, não importando sua idade ou cor. Qual é a probabilidade de que, após2006 dias, a colônia tenha exatamente uma ameba amarela?

Solução: Note que, após $x$ dias, haverá $x$ amebas vermelhas e apenas 1 ameba amarela, logo a probabilidade de uma ameba vermelha se duplicar será de $\frac{x}{x+1}$. Sendo assim, a probabilidade de que a colônia tenha exatamente uma ameba amarela após 2006 dias será de

$$
\frac{1}{2} \cdot \frac{2}{3} \cdot \frac{3}{4} \cdot \ldots \cdot \frac{2006}{2007}=\frac{1}{2007}
$$


Problema Olímpico 6. (Canguru de Matemática - 2017) As faces de quatro dados na forma de tetraedros regulares apresentam os algarismos 2, 0, 1 e 7, um por face. Se os dados são perfeitamente balanceados, qual é a probabilidade de lançarmos todos esses dados e obtermos o número 2017 usando exatamente um algarismo de uma face visível de cada um deles?

Solução: Veja que é bem mais simples calcular a probabilidade de não se obter o número 2017, do que obter. Sendo assim, usaremos a estratégia do complementar. Logo, para não se obter o número 2017 é preciso que nas bases de todos os dados (faces não visíveis) saiam os mesmos números 0 , 1, 2 ou 7. Logo a probabilidade de isto acontecer é de:

$$
\frac{1}{4} \cdot \frac{1}{4} \cdot \frac{1}{4} \cdot \frac{1}{4}=\frac{1}{4^{4}}
$$

Como são quatro números diferentes, a probabilidade de não se obter 2017 é

$$
4 \cdot \frac{1}{4^{4}}=\frac{1}{4^{3}}=\frac{1}{64}
$$

Portanto, probabilidade de se obter o número 2017 é

$$
1-\frac{1}{64}=\frac{63}{64}
$$

Alguns problemas podem ser divididos em casos, com o objetivo de facilitar a resolução e outros porquê não há outra saída. Sendo assim, analisa-se e calcula-se cada um de forma separada e depois soma as probabilidades de cada um dos casos, como veremos nos problemas a seguir.

Problema Olímpico 7. (OBMEP - 2015) Na figura, o círculo das centenas está dividido em três setores, um semicircular e outros dois de mesma área. Cada um dos outros dois círculos estão divididos em setores de mesma área. As setas nesses círculos, quando giradas, param ao acaso em algum setor, determinando um número de três algarismos. Por exemplo, na figura elas determinaram o número 331.

\section{Figura 3: Relógios}

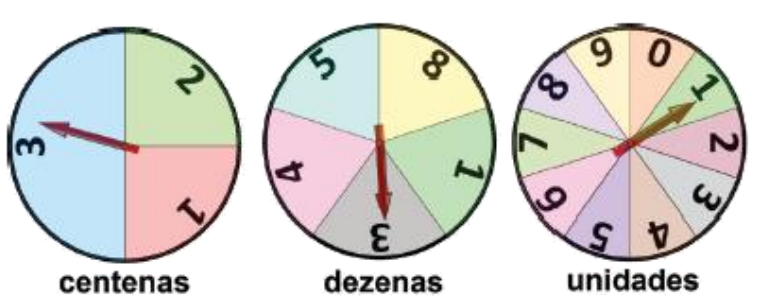

Fonte: OBMEP (2015) 
Qual é a probabilidade de que o número determinado pelas setas, após serem giradas, seja maior do que 260 ?

Solução: Teremos que dividir o problema em dois casos, a saber, quando o ponteiro da roleta das centenas para no número 3 e quando parar no número 2, visto que se parar no número 1 , o resultado final será menor que 260. Daí temos:

$\underline{1^{\circ} \text { Caso }}(\mathrm{O}$ ponteiro da roleta das centenas parar no número 3) Note que a probabilidade do ponteiro parar no número 3 é igual a $\frac{1}{2}$, pois a região que ocupa é metade da roleta. Sendo assim qualquer resultado que sair nas roletas das dezenas e unidades proporcionará um número maior que 260, logo temos:

$$
\frac{1}{2} \cdot 1 \cdot 1=\frac{1}{2}
$$

$\underline{2^{\circ} \text { Caso }}(O$ ponteiro da roleta das centenas parar no numero 2$)$ Note que a probabilidade do ponteiro parar no número 2 é igual a $\frac{1}{4}$ (Se você dividir a região do 3 pela metade obterá quatro regiões iguais), no entanto, na roleta das dezenas apenas um valor (o número 8) fará com que o resultado final seja maior do que 260 , e a probabilidade desse valor sair é de $\frac{1}{5}$. Já na roleta das unidades, qualquer valor será útil. Sendo assim temos:

$$
\frac{1}{4} \cdot \frac{1}{5} \cdot 1=\frac{1}{20}
$$

Portanto, a probabilidade de que o número determinado pelas setas, após serem giradas, seja maior do que 260 é de:

$$
\frac{1}{2}+\frac{1}{20}=\frac{10}{20}+\frac{1}{20}=\frac{11}{20}=0,55 \therefore 55 \%
$$

Problema Olímpico 8. (OBMEP 2014) Dois dados têm suas faces pintadas de vermelho ou azul. Ao jogá-los, a probabilidade de observarmos duas faces superiores de mesma cor é 11/18. Se um deles tem cinco faces vermelhas e uma azul, quantas faces vermelhas tem o outro?

Solução: Teremos que dividir em dois casos, a saber, as duas faces serem vermelhas e as duas faces serem azuis. A princípio, seja $x$ a quantidade de faces vermelhas do segundo dado. Logo,

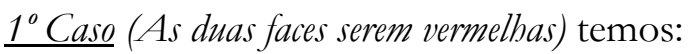

$$
\frac{5}{6} \cdot \frac{x}{6}=\frac{5 x}{36}
$$

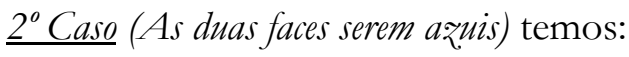




$$
\frac{1}{6} \cdot \frac{(6-x)}{6}=\frac{(6-x)}{36}
$$

Como a probabilidade de sair as duas faces de mesma cor é igual a $\frac{11}{18}$, então:

$$
\frac{5 x}{36}+\frac{(6-x)}{36}=\frac{11}{18} \Leftrightarrow \frac{4 x+6}{2}=11 \Leftrightarrow 4 x+6=22 \Leftrightarrow x=4
$$

Portanto, o outro dado possui 4 faces vermelhas.

Problema Olímpico 9. (Canguru de Matemática - 2015) Amanda tem um dado comum, com os pontos 1,2,3,4,5 e 6 em suas faces. Bruna tem um dado estranho, com os pontos 2, 2, 2, 5 , 5 e 5 em suas faces. Elas combinam lançar os dois dados simultaneamente e quem tirar a maior pontuação é a vencedora. Caso os números sejam iguais, ninguém vence. Qual é a probabilidade de Bruna vencer?

Solução: Vamos analisar duas situações, a primeira é se Bruna tirar o número 2 e a segunda é se Bruna tirar o número 5. Daí temos:

$\underline{1^{\circ} \text { Caso }}$ (Bruna tirar o número 2) A probabilidade de Bruna tirar o número 2 é de $\frac{3}{6}=\frac{1}{2}$, logo de vencer Amanda será:

$$
\frac{1}{2} \cdot \frac{1}{6}=\frac{1}{12}
$$

$\underline{2^{\circ} \text { Caso }}$ (Bruna tirar o número 5) A probabilidade de Bruna tirar o número 5 é de $\frac{3}{6}=\frac{1}{2}$, logo de vencer Amanda será:

$$
\frac{1}{2} \cdot \frac{4}{6}=\frac{4}{12}
$$

Portanto, a probabilidade de vencer Amanda no lançamento dos dados é

$$
\frac{1}{12}+\frac{4}{12}=\frac{5}{12}
$$

Para finalizar, vamos resolver um problema bem interessante, que caiu na OBMEP de 2019, ano passado, e que pode se tornar bem complicado, mas uma ferramenta dos métodos de contagem pouco usada, chamada permutações com elementos repetidos, nos ajudará a resolver de forma simples. Vejamos.

Problema Olímpico 10. (OBMEP - 2019) Uma formiga caminha pela grade abaixo, podendo se mover apenas para a direita ou para cima. Se tiver duas opções para se mover, ela escolhe uma ao 
acaso, com probabilidade 1/2. Qual é a probabilidade de que a formiga comece no ponto A e termine no ponto $\mathrm{B}$ ?

Figura 4: Caminhos

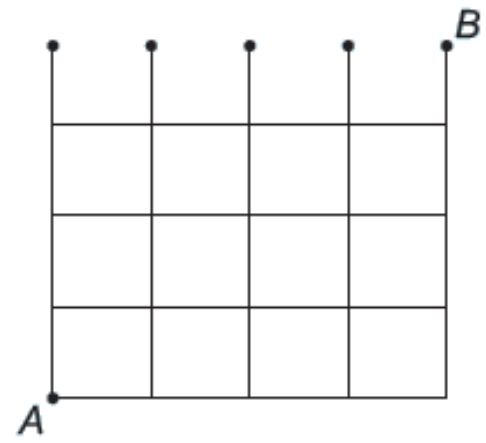

Fonte: OBMEP (2019)

Solução: Vamos fixar os pontos $P 1, P 2, P 3, P 4$ e $B$ que serão nossas "portas" de entrada na chegada como mostra a figura abaixo.

Figura 5: Caminhos com "Portas"

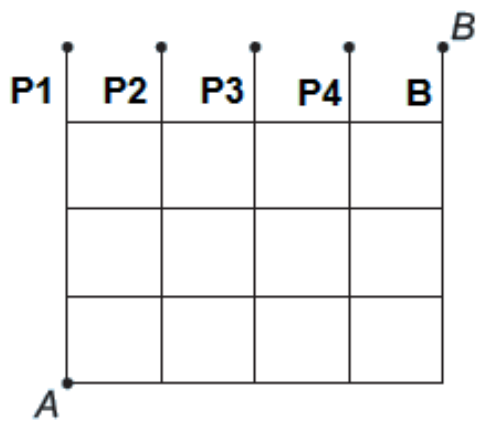

Fonte: Autor ${ }^{5}$

Vejamos os caminhos possíveis.

i) Partindo de A até chegar em P1 temos apenas um caminho.

ii) Partindo de A até chegar em P2, um caminho possível seria DCCC (1 passo a Direita e 3 para Cima), porém não é o único, logo precisamos saber quantas são as permutações possíveis, no entanto há elementos repetidos, então temos que calcular quantas são as permutações com elementos repetidos, ou seja,

$$
P_{4}^{3,1}=\frac{4 !}{3 ! \cdot 1 !}=4
$$

iii) Partindo de A até P3, um caminho possível seria DDCCC. De forma análoga temos

$$
P_{5}^{3,2}=\frac{5 !}{3 ! \cdot 2 !}=10
$$

\footnotetext{
${ }^{5}$ Pequena adaptação pelo autor da figura 4 para ajudar na compreensão da resolução do problema.
} 
iv) Partindo de A até P4, um caminho alternativo seria DDDCCC. De forma análoga temos

$$
P_{6}^{3,3}=\frac{6 !}{3 ! \cdot 3 !}=20 \text {. }
$$

v) E por fim, partindo de A até B, um caminho alternativo seria DDDDCCC. De forma análoga temos

$$
P_{7}^{4,3}=\frac{7 !}{4 ! \cdot 3 !}=35
$$

Logo, a probabilidade de que a formiga comece no ponto A e termine no ponto B é:

$$
\frac{P_{7}^{4,3}}{1+P_{4}^{3,1}+P_{5}^{3,2}+P_{6}^{3,3}+P_{7}^{4,3}}=\frac{35}{1+4+10+20+35}=\frac{35}{70}=\frac{1}{2} .
$$

\section{CONSIDERAÇÕES FINAIS}

A probabilidade proporciona a possibilidade de prevê as chances de um determinado acontecimento ocorrer de um experimento aleatório, o que nos mostra a atratividade de trabalhar com possíveis previsões, como por exemplo, a chance de sair um número par no lançamento de um dado convencional não viciado e até mesmo trabalhar as chances de se ganhar na loteria, ou em um simples jogo de azar. O que não falta são possibilidades de se ensinar de forma divertir, como usar problemas instigante que provoque os alunos a quererem buscar as respostas, além de criar diversas estratégias para as resoluções.

A probabilidade pode ser apresentada de forma divertida para os alunos, se for utilizado de forma chamativa e intrigante, provocando os alunos a quererem resolver os problemas, e a utilização de problemas olímpicos é uma forma de fazer isso.

Com este trabalho acredita-se que foi possível mostrar a importância da utilização de problemas olímpicos para o ensino de probabilidade na educação básica, melhorando assim, significativamente o ensino-aprendizagem, além de motivar os alunos a participar destas competições cada vez mais.

Existem outros tópicos de matemática que podem ser utilizados problemas olímpicos para serem ensinados de forma mais provocativa e divertida, como áreas, funções, geometria e etc. $\mathrm{O}$ que se pode notar é que a utilização de problemas olímpicos é uma ferramenta bem instigante e importante para ser utilizada. 


\section{REFERÊNCIAS}

[1] BALESTRI, R. Matemática: Interação e Tecnologia Vol.2. 2º ed. São Paulo: Leya, 2016.

[2] BRASIL. Concurso Canguru de Matemática Brasil. Canguru de Matemática 2020.

Disponívelem: $<$ https://www.cangurudematematicabrasil.com.br/para-escolas/provasanteriores >. Acessado em: 05 de jan. 2020.

[3] BRASIL. Olimpíada Brasileira de Matemática. OBM 2019. Rio de Janeiro, 2019. Disponível em: < https://www.obm.org.br/como-se-preparar/provas-e-gabaritos/>. Acessado em: 05 de jan. 2020.

[4] BRASIL. Olimpíada Brasileira de Matemática das Escolas Públicas. OBMEP 2019. Rio de Janeiro, 2019. Disponível em: <http://www.obmep.org.br/provas.htm>. Acessado em: 05 de jan. 2020.

[5] CARVALHO, P.C. P. Métodos de Contagem e Probabilidade. Rio de Janeiro: IMPA, 2015.

[6] FREITAS, J.L. M. de. Et. al. Fundamentos e metodologias para o ciclo inicial. In: VIII Encontro Nacional de Educação Matemática (ENEM), 2004, Pernambuco, Anais - Minicurso. Pernambuco: SBEM, 2004. Disponível em

$<$ http://www.sbembrasil.org.br/files/viii/pdf/01/MC74619047872.pdf>. Acesso em 8 de jan. de 2020 .

[7] GOMES, A. M. S.; SOUZA, R. A. DE. O Princípio da Inclusão - Exclusão e as Permutacões Caóticas: Métodos Alternativos de Contagem. Revista Científica Multidisciplinar Núcleo do Conhecimento, v. 16, p. 193-209, 2018.

[8] IEZZI, G. et. al. Matemática: Ciência e Aplicaşões Vol.2. 9º ed. São Paulo: Saraiva,2016.

[9] MORGADO, A. C.; et. Al. Análise combinatória e probabilidade. 9 ed, Rio de Janeiro: SBM, 1991.

[10] OLIVEIRA, K.I.M.; FERNÁNDEZ, A.J.C. Iniciação à Matemática: Um Curso com Problemas e Soluções. 2. ed. Rio de Janeiro: SBM, 2012.

[11] PIAUÍ. Olimpíada Piauiense de Matemática. OPIM 2019. Piauí, 2019. Disponível em: <http://opim.ufpi.br/>. Acessado em: 8 de jan. 2019. 